\title{
Thiosemicarbazone Derivatives as Inhibitors of Amyloid- $\beta$ Aggregation: Effect of Metal Coordination
}

\author{
Ana I. Matesanz, ${ }^{+}$Ana B. Caballero, ${ }^{\ddagger}$ Carmen Lorenzo, ${ }^{+\nabla}$ Alba Espargaró,, sll Raimon Sabaté,,$\|$ \\ Adoración G. Quiroga ${ }^{* \#+}$ and Patrick Gamez ${ }^{* \neq \| \perp}$ \\ † Department of Inorganic Chemistry (M-07), School of Sciences, Universidad Autónoma de \\ Madrid, 28049-Madrid, Spain. E-mail: adoracion.gomez@uam.es \\ \# Institute for Advanced Research in Chemical Sciences (IAdChem), Universidad Autónoma de \\ Madrid, Madrid-28049, Spain \\ ‡ nanoBIC, Departament de Química Inorgànica i Orgànica, Facultat de Química, Universitat de \\ Barcelona, Martí i Franquès, 1-11, 08028 Barcelona, Spain. E-mail: patrick.gamez@qi.ub.es \\ \|I Institute of Nanoscience and Nanotechnology (IN²UB), Universitat de Barcelona, 08028 \\ Barcelona, Spain.
}

$\S$ Departament de Fisicoquímica, Facultat de Farmàcia i Ciències de l'Alimentació, Universitat de Barcelona, Avda. Joan XXIII 27-31, 08028 Barcelona, Spain.

${ }^{\perp}$ Catalan Institution for Research and Advanced Studies, Passeig Lluís Companys 23, 08010 Barcelona, Spain. 
Table S1. Crystal data and structure refinement for compounds $\mathbf{2}$ and $\mathbf{3}$.

\begin{tabular}{|c|c|c|}
\hline Compound & 2 & 3 \\
\hline Empirical formula & $\mathrm{C}_{28} \mathrm{H}_{50} \mathrm{~N}_{8} \mathrm{O}_{4} \mathrm{PdS}_{6}$ & $\mathrm{C}_{22} \mathrm{H}_{30} \mathrm{~N}_{8} \mathrm{PdS}_{2}$ \\
\hline Formula weight (g mol${ }^{-1}$ ) & 861.52 & 577.06 \\
\hline Temperature (K) & $296(2)$ & $293(2)$ \\
\hline Crystal system & monoclinic & triclinic \\
\hline Space group & $P 2_{1} / \mathrm{c}$ & $P-1$ \\
\hline Crystal size $\left(\mathrm{mm}^{3}\right)$ & $0.21 \times 0.08 \times 0.08$ & $0.16 \times 0.04 \times 0.02$ \\
\hline$a(\AA)$ & $10.4283(3)$ & $9.4991(6)$ \\
\hline$b(\AA)$ & $28.3190(9)$ & $10.9920(7)$ \\
\hline$c(\AA)$ & $7.0820(3)$ & $12.2666(8)$ \\
\hline$\alpha\left(^{\circ}\right)$ & 90 & $94.182(4)$ \\
\hline$B\left({ }^{\circ}\right)$ & $108.764(2)$ & $99.319(3)$ \\
\hline$\gamma\left(^{\circ}\right)$ & 90 & $93.672(4)$ \\
\hline$V\left(\AA^{3}\right)$ & $1980.29(12)$ & $1256.79(14)$ \\
\hline$Z$ & 2 & 2 \\
\hline$\rho_{\text {calcd }}$ & 1.445 & 1.525 \\
\hline$\mu\left(\mathrm{mm}^{-1}\right)$ & 0.827 & 0.931 \\
\hline$F(000)$ & 896 & 592 \\
\hline$\vartheta$ for data collection $\left({ }^{\circ}\right)$ & $1.44-25.35$ & $2.62-18.01$ \\
\hline Reflections collected / unique & $29360 / 3636$ & 20065 / 4482 \\
\hline Completeness to theta & 0.999 & 0.974 \\
\hline Data / restraints / parameters & $3636 / 0$ / 220 & 4482 / 0 / 307 \\
\hline Goodness-of-fit on $F^{2}$ & 1.115 & 1.025 \\
\hline Final $R$ indices $[1>2 \sigma(I)]$ & $R 1=0.0556, w R 2=0.1491$ & $\mathrm{R} 1=0.0488, w R 2=0.1115$ \\
\hline$R$ indices (all data) & $\mathrm{R} 1=0.0842, \mathrm{wR} 2=0.1773$ & $\mathrm{R} 1=0.1167, \mathrm{wR} 2=0.1582$ \\
\hline Largest diff. peak and hole $\left(e \AA^{3}\right)$ & 2.751 and -0.783 & 0.501 and -0.895 \\
\hline
\end{tabular}


Table S2. Selected bond distances ( $(\AA)$ and angles (ㅇ) for complex $\operatorname{Pd}\left(\mathbf{L}^{2}\right)_{2}(\mathbf{2})$.

\begin{tabular}{|c|c||c|c|}
\hline \multicolumn{2}{|c||}{ Bond lengths } & \multicolumn{2}{c|}{ Angles } \\
\hline $\mathrm{Pd}_{1}-\mathrm{S}_{1}$ & $2.2879(17)$ & $\mathrm{N}_{1}-\mathrm{Pd}_{1}-\mathrm{S}_{1}$ & $82.61(15)$ \\
\hline $\mathrm{Pd}_{1}-\mathrm{N}_{1}$ & $2.031(5)$ & $\mathrm{N}_{1}-\mathrm{Pd}_{1}-\mathrm{S}_{1} \mathrm{a}$ & $97.39(15)$ \\
\hline $\mathrm{C}_{1}-\mathrm{S}_{1}$ & $1.750(6)$ & $\mathrm{N}_{1}-\mathrm{Pd}_{1}-\mathrm{N}_{1} \mathrm{a}$ & 180 \\
\hline $\mathrm{C}_{1}-\mathrm{N}_{2}$ & $1.301(8)$ & $\mathrm{S}_{1}-\mathrm{Pd}_{1}-\mathrm{S}_{1} \mathrm{a}$ & 180 \\
\hline $\mathrm{C}_{2}-\mathrm{N}_{1}$ & $1.301(8)$ & \multicolumn{2}{|}{} \\
\hline $\mathrm{N}_{1}-\mathrm{N}_{2}$ & $1.383(7)$ & & \\
\hline
\end{tabular}

Symmetry operation: $a=1-x, 1-y, 1-z$

Table S3. Selected bond distances $(\AA ̊)$ and angles (ㅇ) for complex $\operatorname{Pd}\left(\mathbf{L}^{3}\right)_{2}(\mathbf{3})$.

\begin{tabular}{|c|c||c|c|}
\hline \multicolumn{2}{|c||}{ Bond lengths } & \multicolumn{2}{c|}{ Angles } \\
\hline $\mathrm{Pd}_{1}-\mathrm{S}_{1}$ & $2.288(2)$ & $\mathrm{N}_{1}-\mathrm{Pd}_{1}-\mathrm{S}_{1}$ & $82.64(17)$ \\
\hline $\mathrm{Pd}_{1}-\mathrm{N}_{1}$ & $2.050(6)$ & $\mathrm{N}_{1}-\mathrm{Pd}_{1}-\mathrm{S}_{1} \mathrm{a}$ & $97.36(17)$ \\
\hline $\mathrm{C}_{1}-\mathrm{S}_{1}$ & $1.754(7)$ & $\mathrm{N}_{1}-\mathrm{Pd}_{1}-\mathrm{N}_{1} \mathrm{a}$ & 180 \\
\hline $\mathrm{C}_{1}-\mathrm{N}_{2}$ & $1.296(9)$ & $\mathrm{S}_{1}-\mathrm{Pd}_{1}-\mathrm{S}_{1} \mathrm{a}$ & 180 \\
\hline $\mathrm{C}_{3}-\mathrm{N}_{1}$ & $1.308(9)$ & & \\
\hline $\mathrm{N}_{1}-\mathrm{N}_{2}$ & $1.391(8)$ & & \\
\end{tabular}

Symmetry operation: $a=-x, 1-y,-z$ 

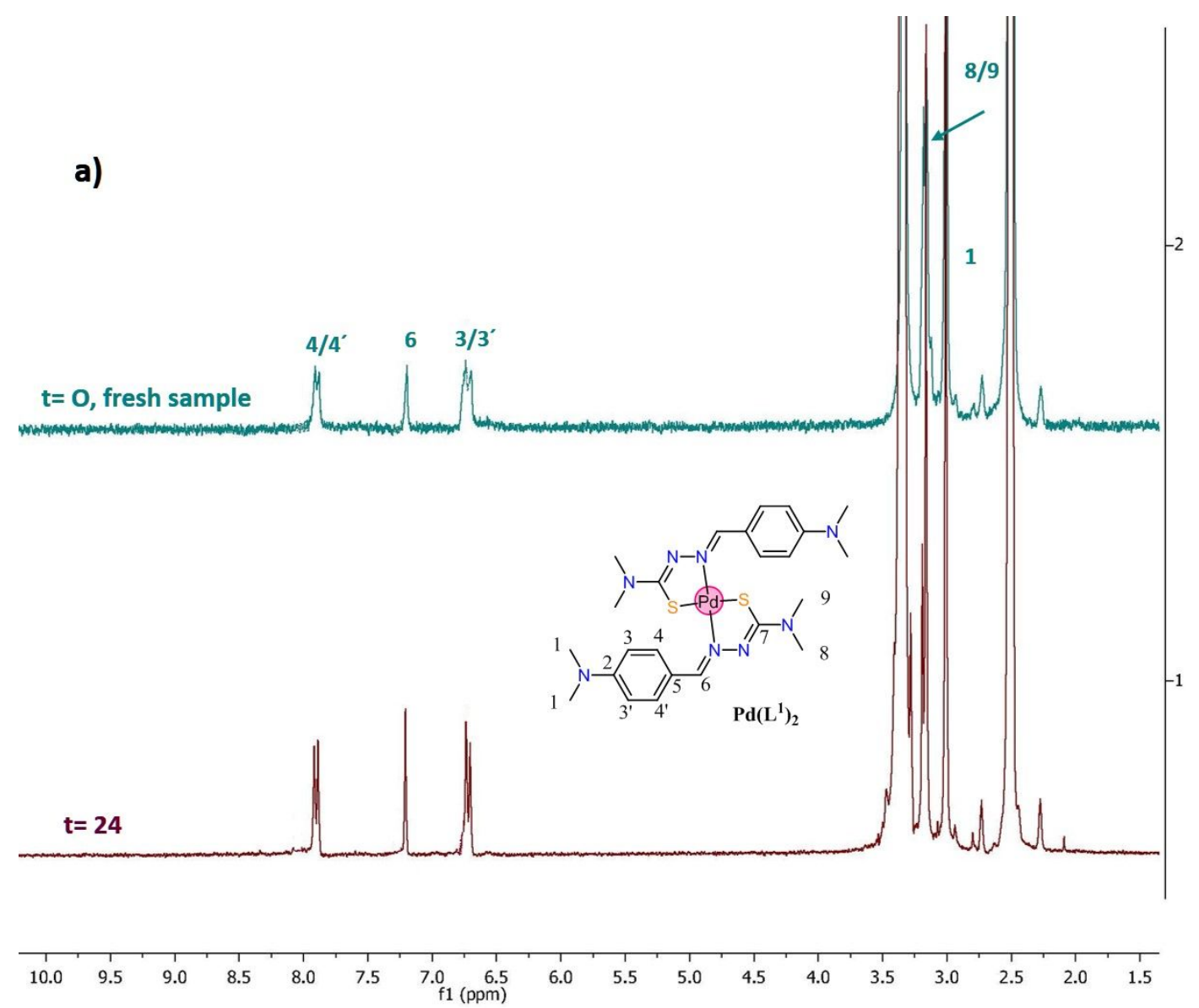

b)

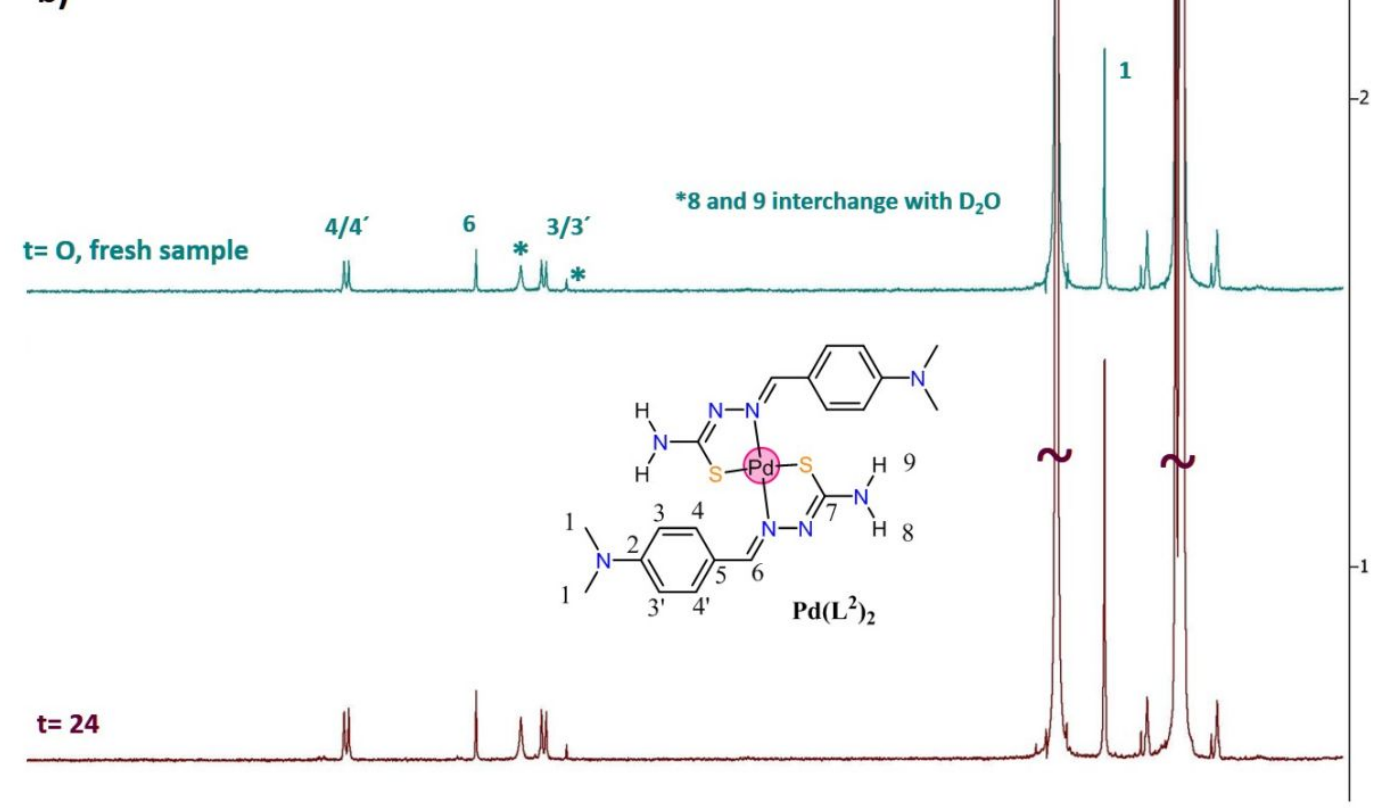

\begin{tabular}{lllllllllllllllll}
\hline & 1 \\
3.0 & 9.5 & 9.0 & 8.5 & 8.0 & 7.5 & 7.0 & $\underset{\mathrm{f} 1(\mathrm{ppm})}{6.0}$ & 5.5 & 5.0 & 4.5 & 4.0 & 3.5 & 3.0 & 2.5 & 2.0 & 1.5
\end{tabular} 


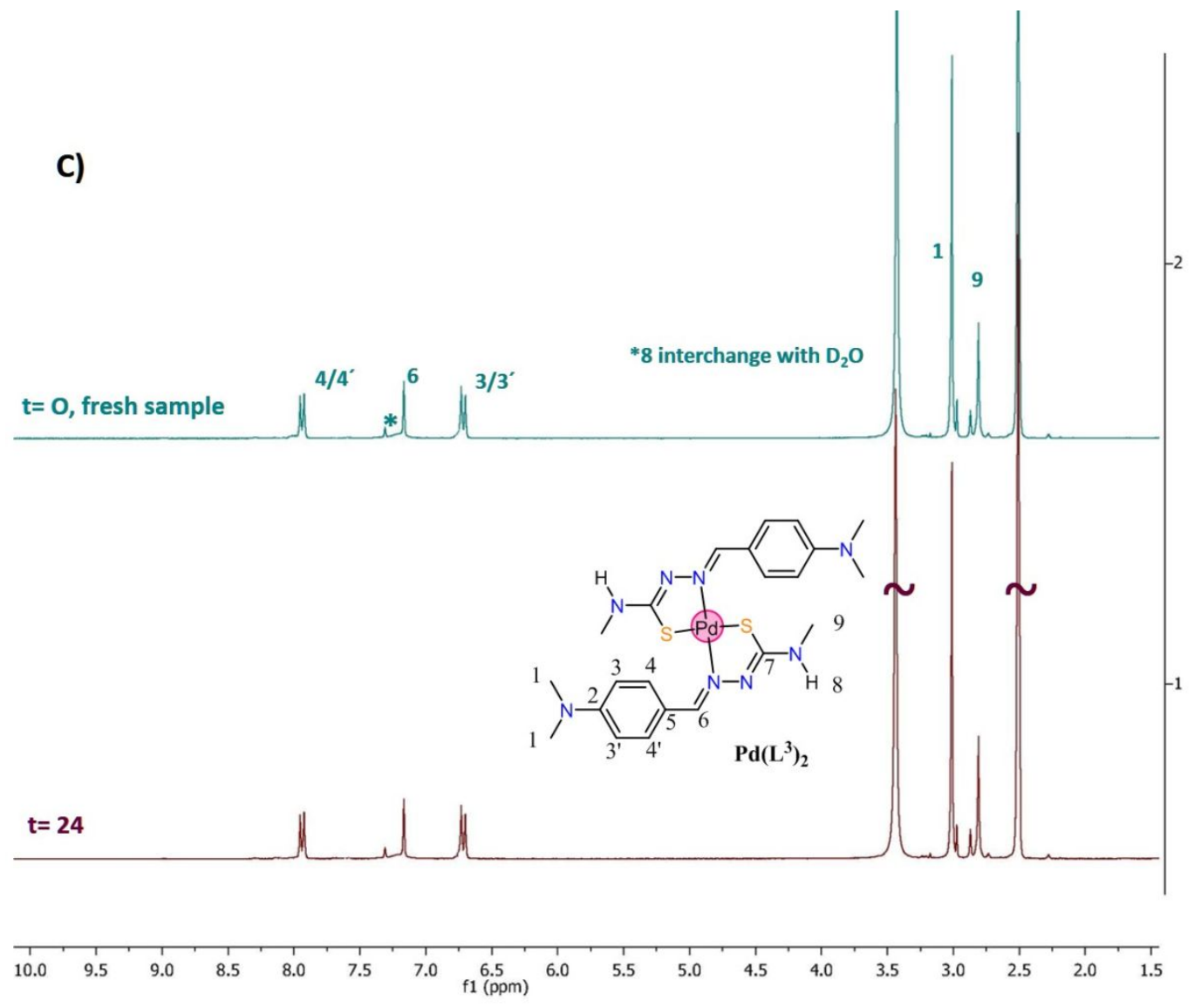

Figure S1. ${ }^{1} \mathrm{H}$ NMR spectra for the complexes in DMSO- $\mathrm{d}_{6}$ fresh and $24 \mathrm{~h}$. 

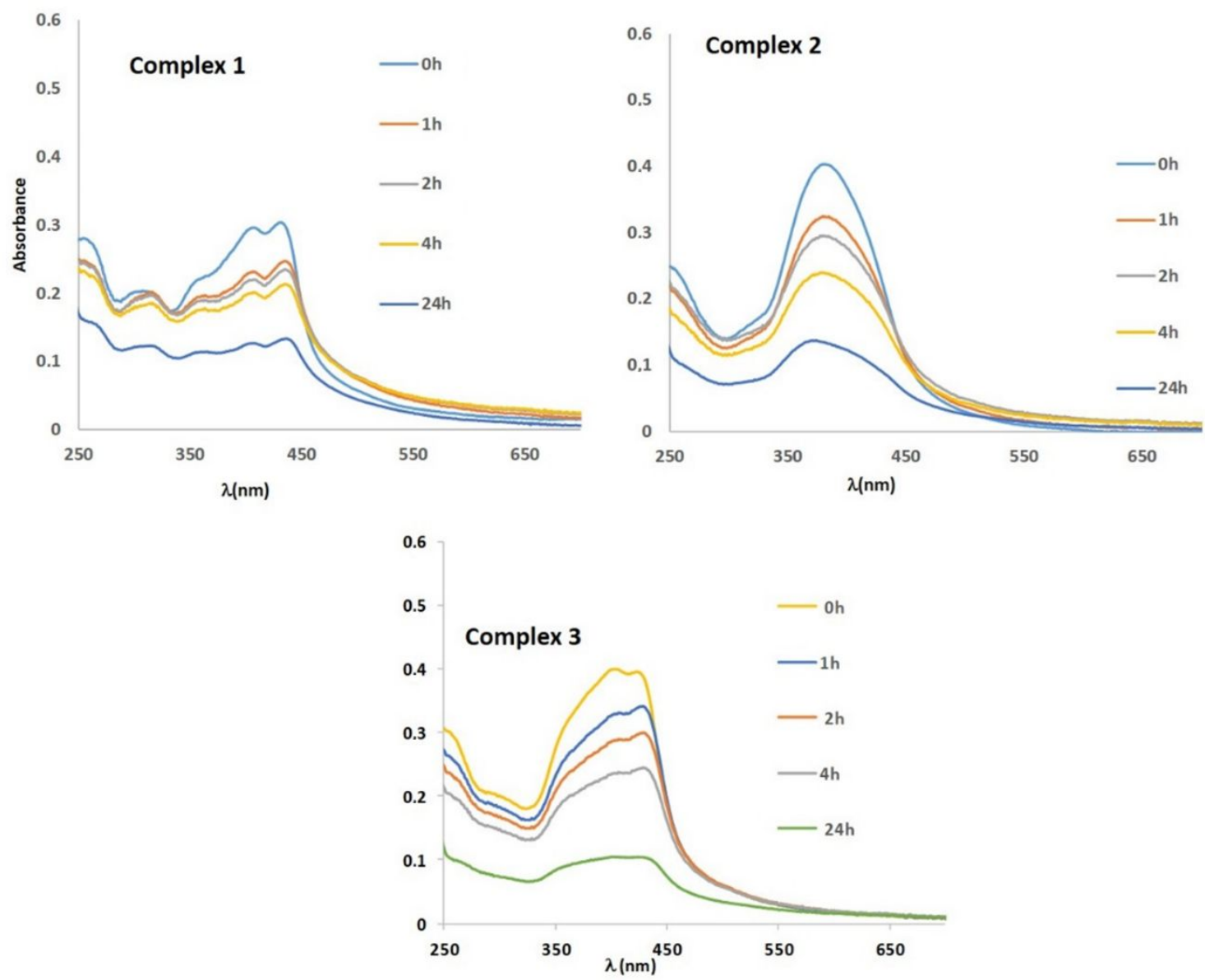

Figure S2. UV-Visible spectra for complexes 1-3 in Tris- $\mathrm{HCl}$ containing 5\% DMSO during $24 \mathrm{~h}$. 


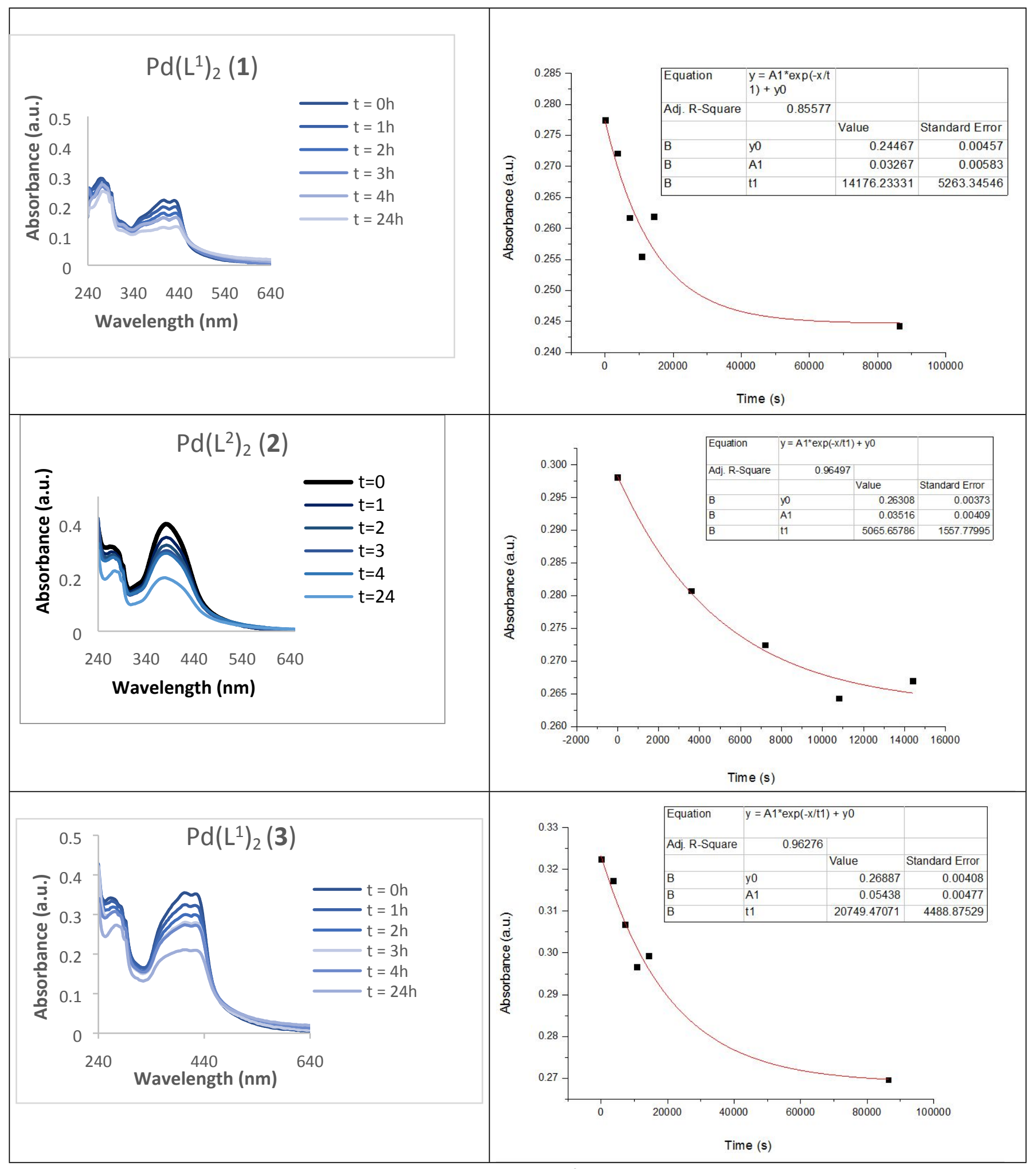

Figure S3. UV-vis spectra illustrating the interaction of complexes 1, $\mathbf{2}$ and $\mathbf{3}$ with lysozyme as a function of time (during $24 \mathrm{~h}$ ). Metal-to-enzyme ratio of 3:1. 


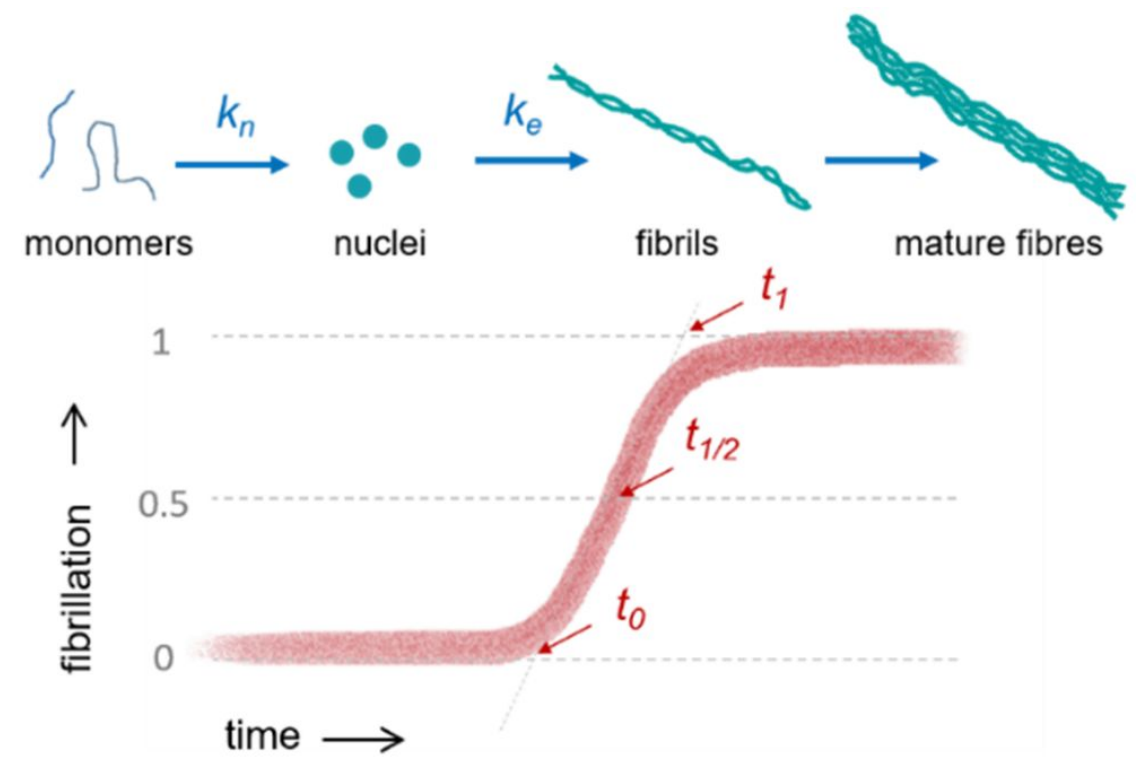

Scheme S1. Schematic representation of $A \beta$ fibrillation, from the nucleation $\left(k_{n}\right)$ to the elongation $\left(k_{\mathrm{e}}\right) \cdot t_{0}=$ lag time before the formation of nuclei; $t_{1 / 2}=$ time required to aggregate half of the protein to its amyloid conformation; $t_{1}=$ time corresponding to the completion of the fibrillation process. 


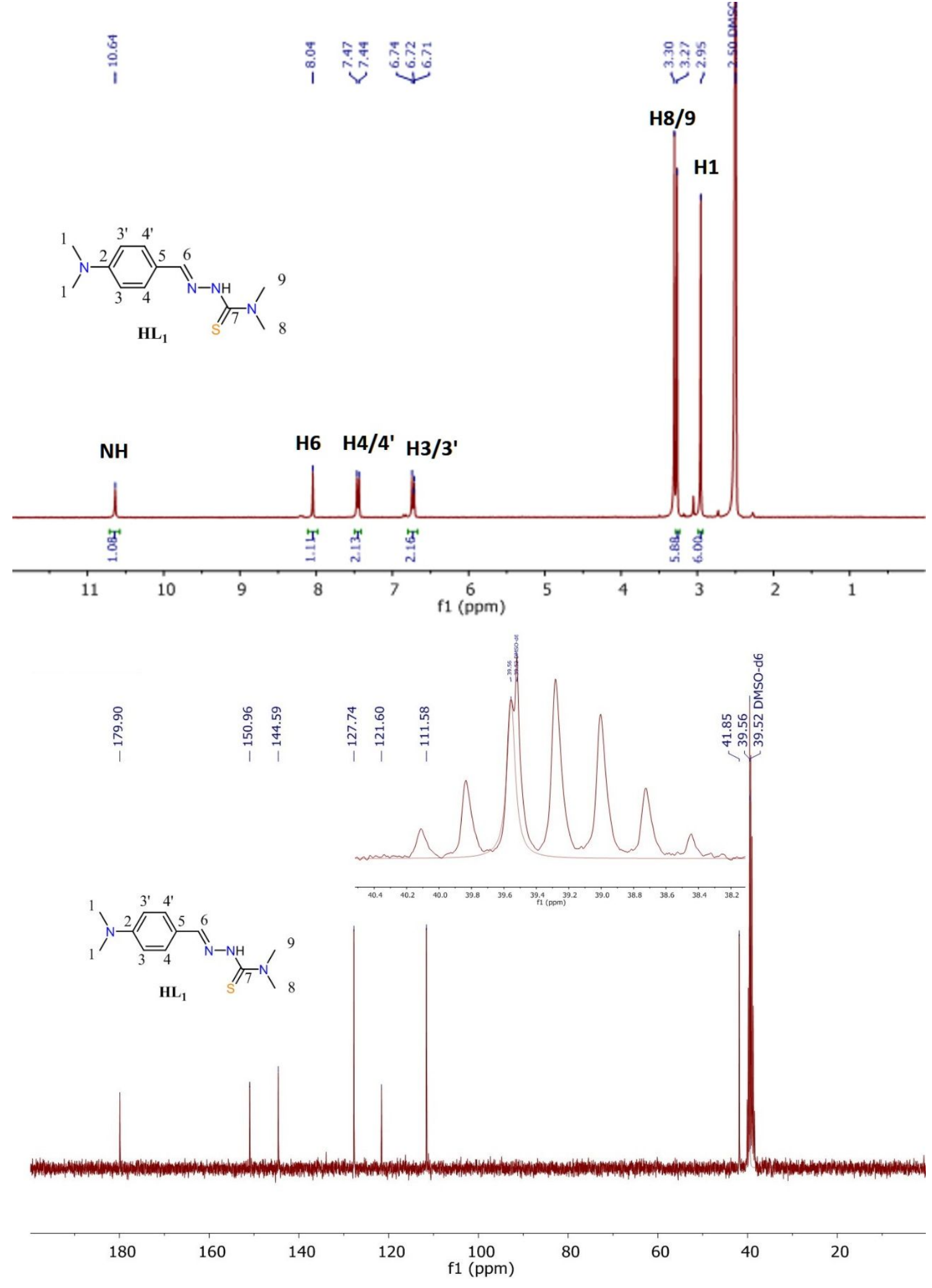

Figure S4. ${ }^{1} \mathrm{H}$ and ${ }^{13} \mathrm{C}$ NMR spectra of ligand $\mathrm{HL1}$ in DMSO- $\mathrm{d}_{6}$. 


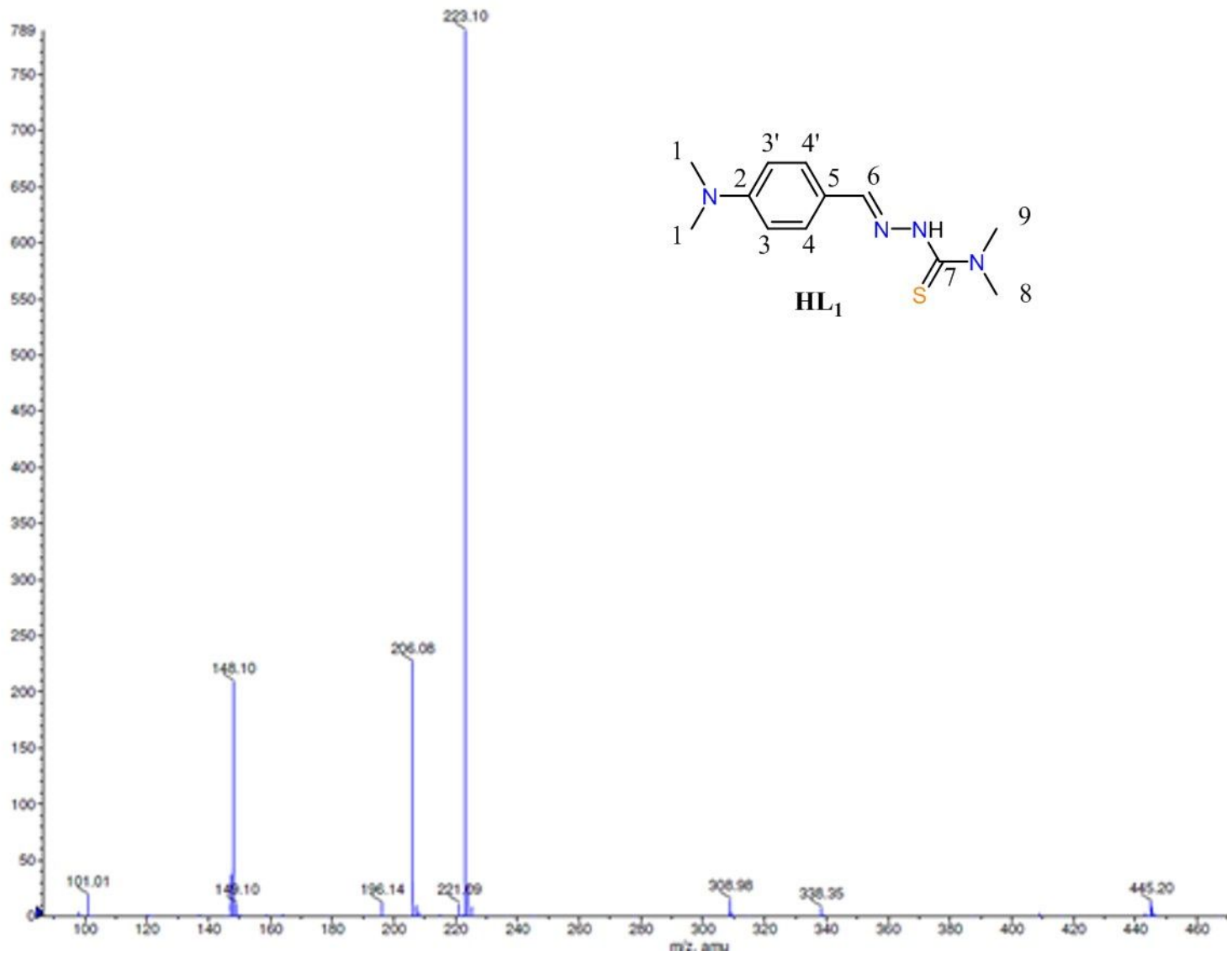

Figure S5. Mass spectrum of ligand HL1.

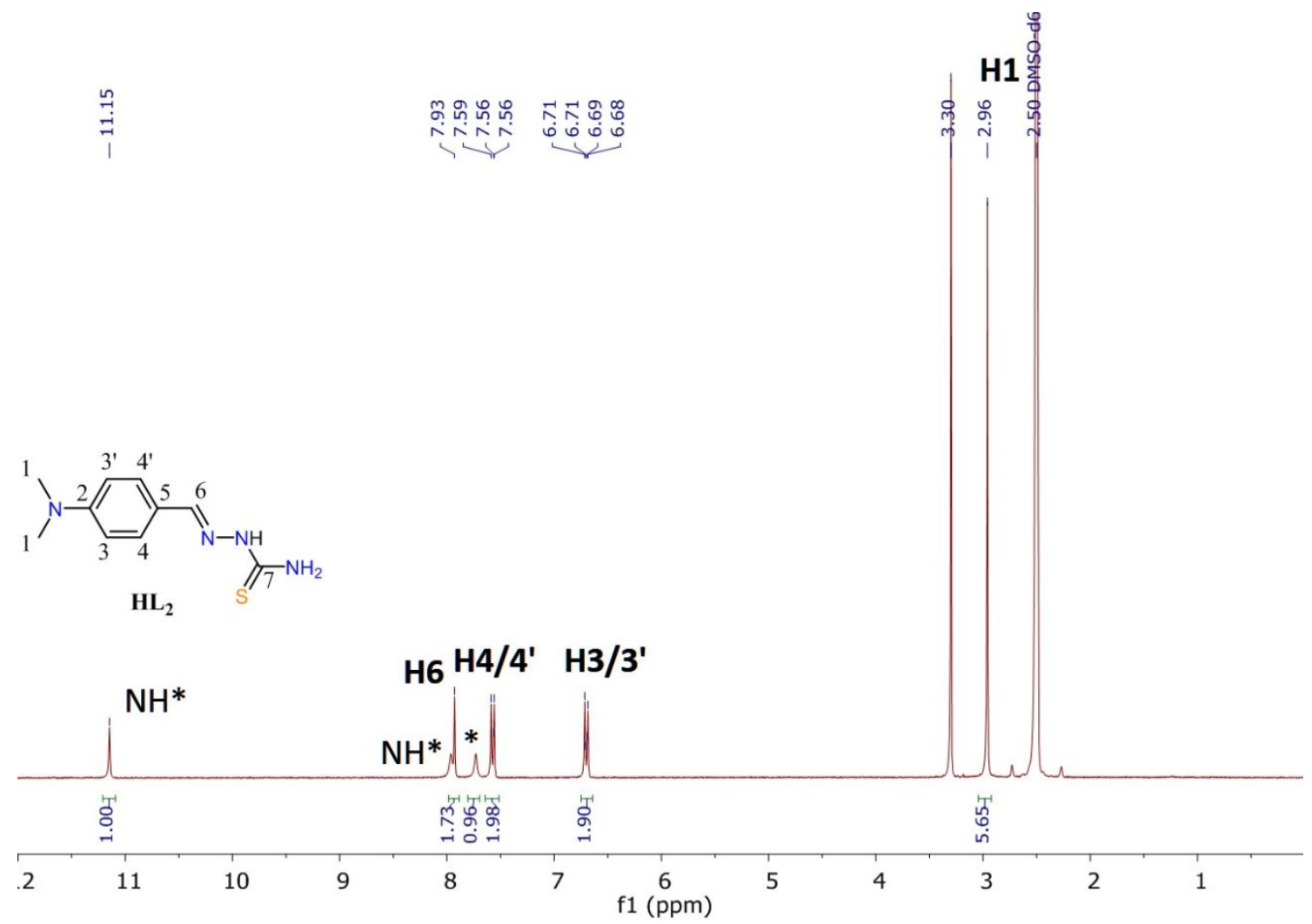

Figure S6. ${ }^{1} \mathrm{H}$ NMR spectrum of ligand $\mathbf{H L 2}$ in DMSO- $\mathrm{d}_{6}$. 


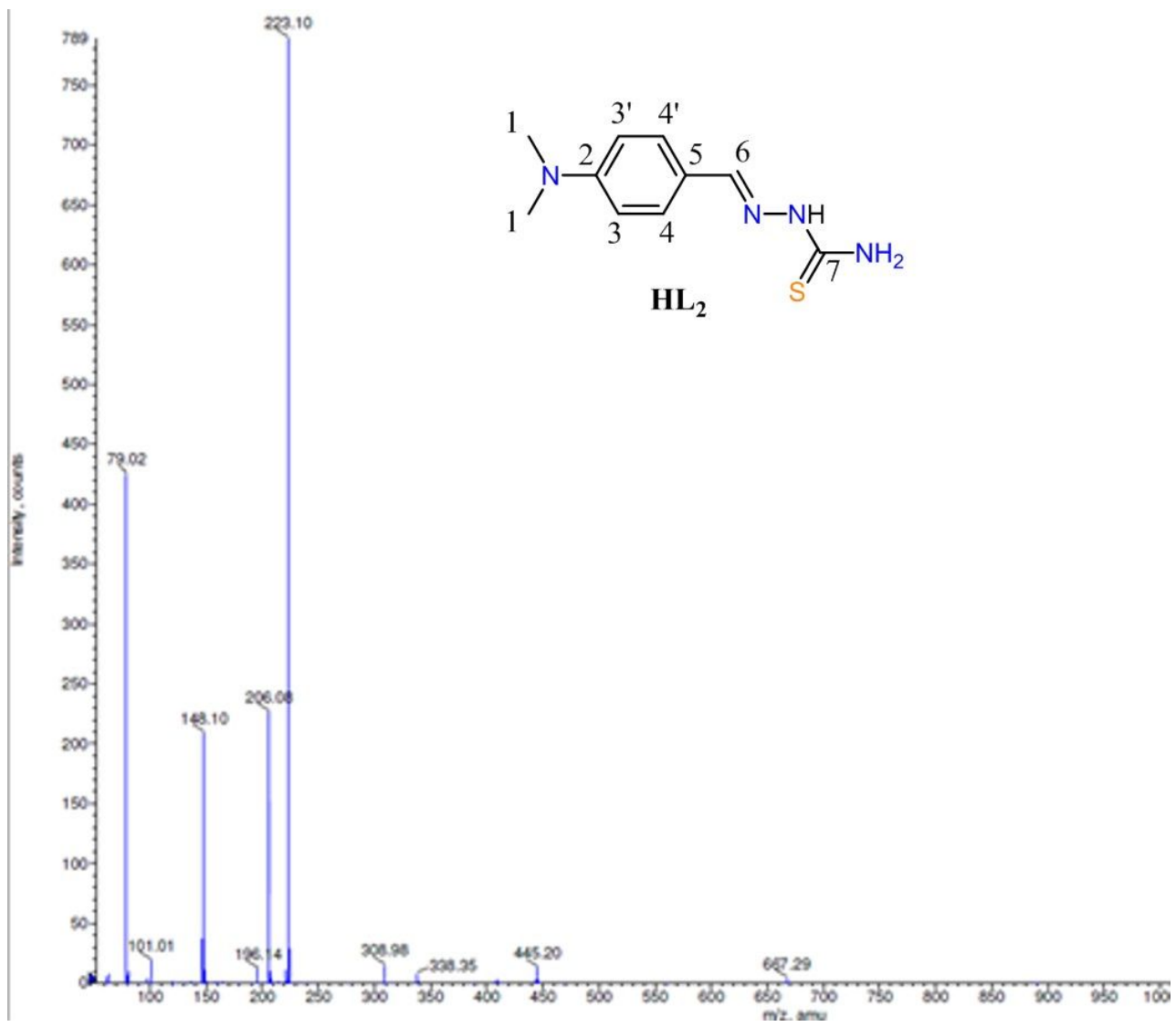

Figure S7. Mass spectrum of ligand HL2.

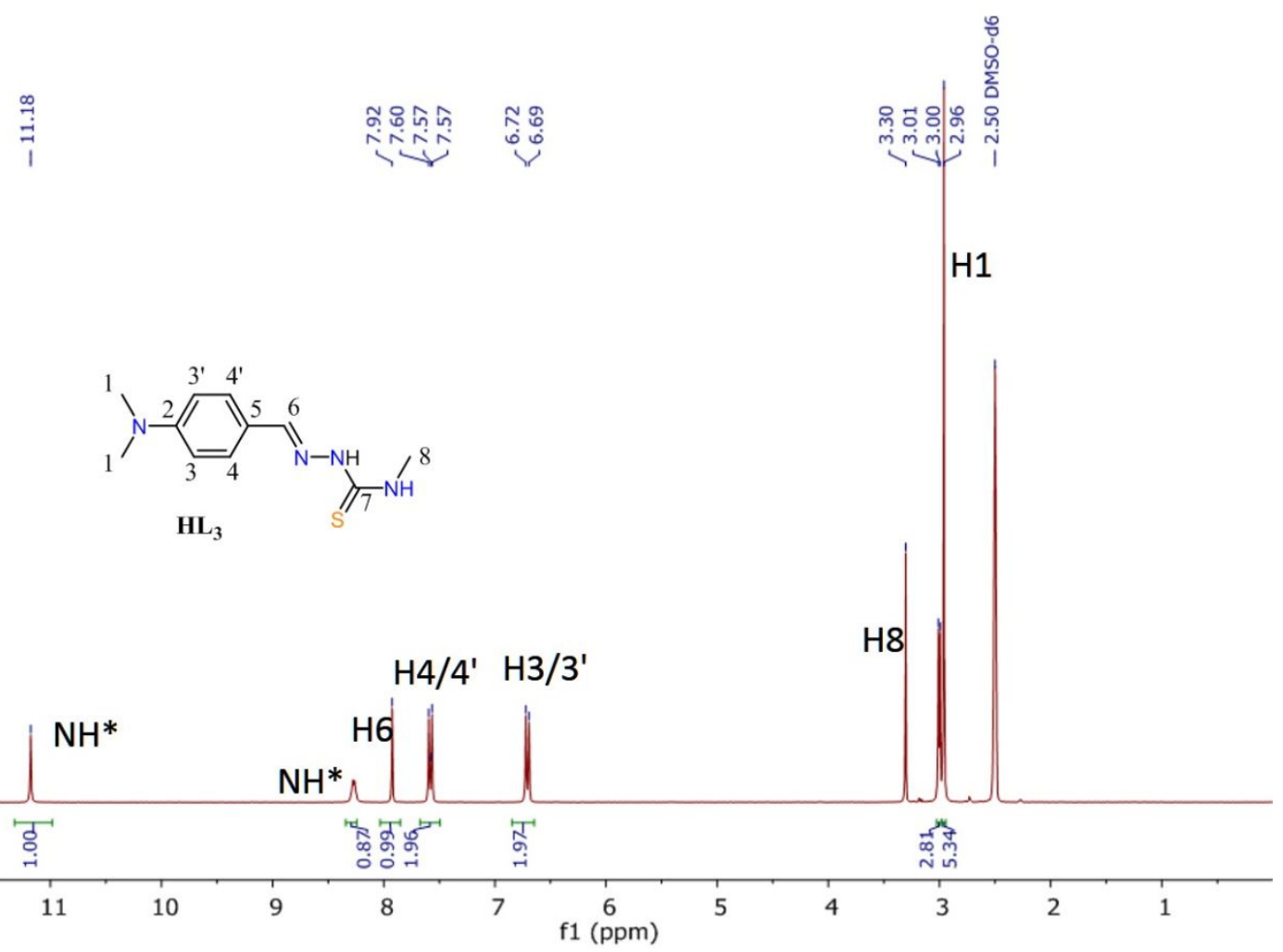




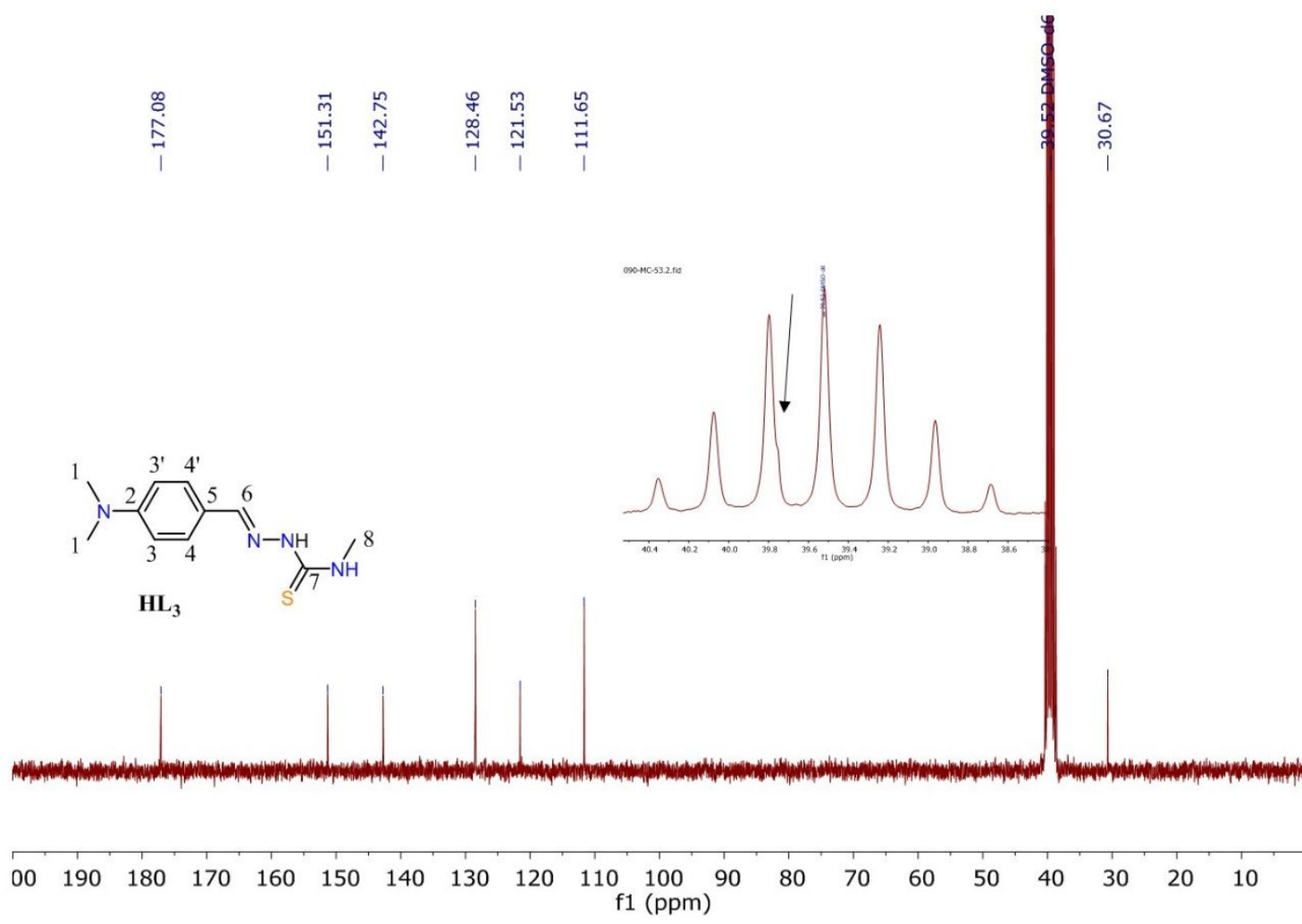

Figure S8. ${ }^{1} \mathrm{H}$ and ${ }^{13} \mathrm{C}$ NMR spectra of ligand $\mathrm{HL3}$ in DMSO- $\mathrm{d}_{6}$.

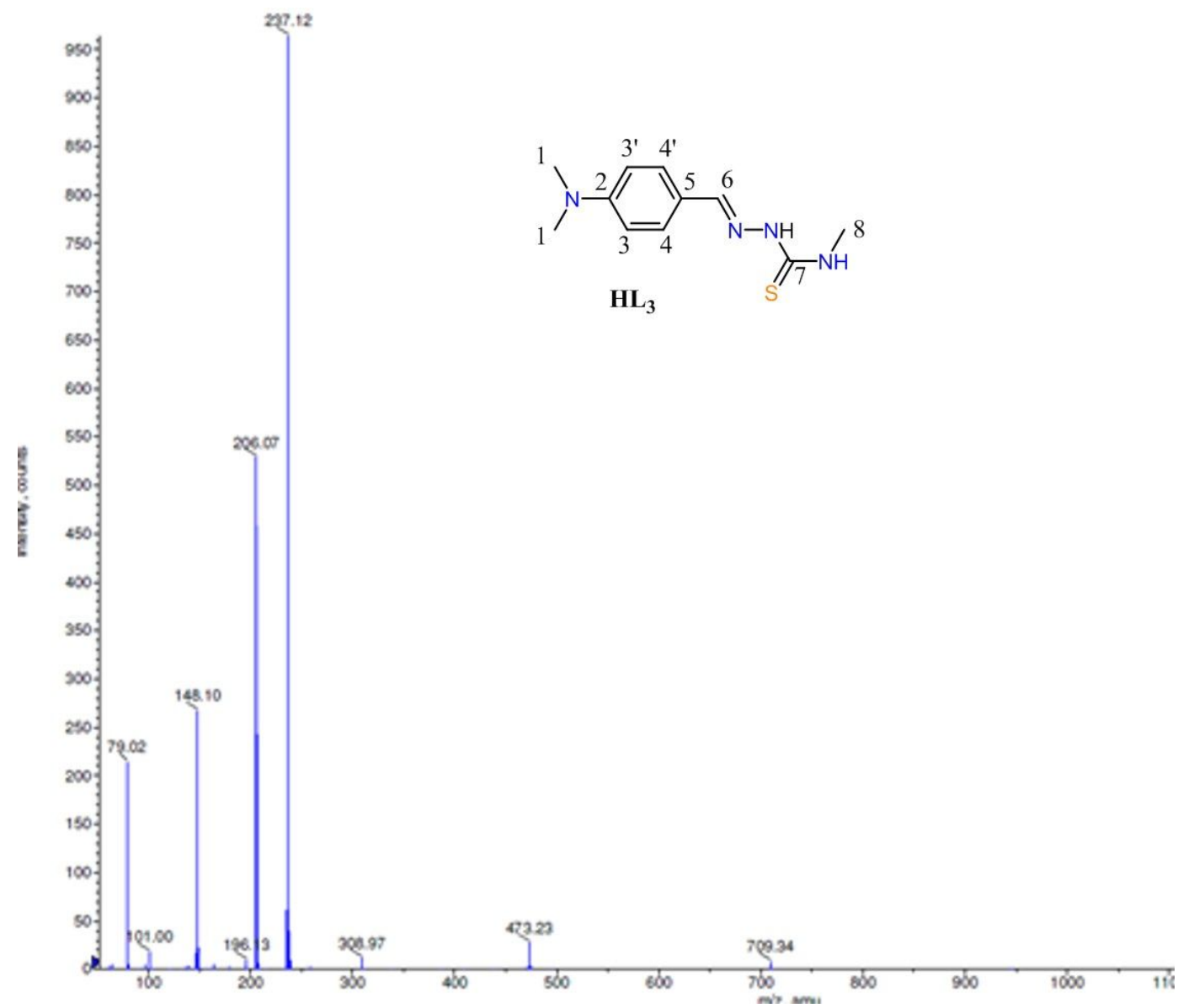

Figure S9. Mass spectrum of ligand HL3. 